CERN-PH-TH/2005-218

SWAT $/ 05 / 448$

\title{
Quark Condensate in Massless QCD from Planar Equivalence
}

\author{
Adi Armoni ${ }^{a}$, Graham Shore ${ }^{a}$ and Gabriele Veneziano ${ }^{b, c}$ \\ ${ }^{a}$ Department of Physics, University of Wales Swansea, \\ Singleton Park, Swansea, SA2 8PP, UK \\ $b$ Theory Division, CERN \\ CH-1211 Geneva 23, Switzerland \\ ${ }^{c}$ Collège de France, 11 place M. Berthelot, 75005 Paris, France
}

\begin{abstract}
A previous estimate of the quark condensate in one-flavour massless QCD from the known value of the gluino condensate in super Yang-Mills (SYM) theory is extended to $N_{f}>1$ by considering the large- $N$ limit of an $S U(N)$ gauge theory with one flavour in the antisymmetric representation and $n_{f}=N_{f}-1$ extra flavours in the fundamental representation. We argue that, even at $n_{f} \neq 0$, suitably chosen correlators in this theory can be mapped to those of SYM as $N \rightarrow \infty$. We give arguments why this correspondence should be particularly good for $N_{f}=3$ and compare our prediction with available (real and Monte Carlo) data.
\end{abstract}




\section{Introduction}

There is overwhelming evidence that QCD is the correct theory of strong interactions. The validity of QCD is confirmed by comparing with data its predictions for short distance (hard) processes, where perturbative calculations can be reliably performed. On the other hand, it is very difficult to carry out reliable analytic calculations in the non-perturbative large-distance regime. In supersymmetric theories the situation is much better, due to holomorphy. In particular the gluino condensate [1] can be evaluated exactly in $\mathcal{N}=1$ super Yang-Mills (referred to in the following as SYM theory)

A while ago, a large- $N$ limit $[2,3]$ that enabled to calculate the quark condensate in one-flavour QCD [4] was suggested. The results were summarized in the review paper [5] (where new results and new ideas are included as well). The idea is to view the fundamental representation of the $S U(3)$ gauge theory as a two-index antisymmetric representation. The next step was to generalize to $S U(N)$ and then to take the large- $N$ limit. At large $N$, the theory with an antisymmetric fermion was shown to be equivalent to SYM in its bosonic sector. Since the exact value of the gluino condensate in known, it was possible to copy its value to the non-supersymmetric theory under consideration. Therefore, up to $1 / N$ corrections (of order $30 \%$ ) it was possible to estimate the value of the gluino condensate in one-flavour QCD.

Following this development, several other works that concern the orientifold large- $N$ equivalence were published. In $[6,7,8]$ the emphasis was put on phenomenological aspects and in $[9,10]$ on the relation with string theory.

The purpose of this letter is to generalize the previous analysis to multiflavour QCD and in particular to discuss massless three-flavour QCD. The idea is the following. Consider an $S U(N)$ gauge theory with one Dirac fermion in the two index antisymmetric representation and $n_{f}$ additional Dirac fermions in the fundamental representation. We name this theory QCD-OR'. For $N=3$, QCD-OR' becomes multi-flavour QCD with $N_{f}=$ $n_{f}+1$ fundamental flavours. This model, when $n_{f}=2$, was considered in the past [11], with a different motivation. When $N \rightarrow \infty$ (while $n_{f}$ is kept fixed) we can neglect the extra fundamental flavours inside the loops and the theory becomes again supersymmetric in its bosonic sector.

The large- $N$ prediction for the quark condensate of multi-flavour QCD coincides, therefore, with the gluino condensate of $\mathcal{N}=1$ SYM. However, while both one-flavour and multi-flavour QCD approach $\mathcal{N}=1 \mathrm{SYM}$ at 
large $N$, the $1 / N$ corrections are different in the two cases. Although we cannot fully control these corrections, we will do our best to estimate them. Moreover, we will present arguments that, in a suitable sector, three-flavour QCD may be even closer to SYM than one-flavour QCD.

The manuscript is organized as follows: in section two we will discuss the planar equivalence of $\mathrm{QCD}-\mathrm{OR}^{\prime}$ and $\mathrm{SYM}$ at large- $N$. In section three we will discuss the issue of how to match the gluino condensate with an appropriate condensate of QCD-OR'. In section four we will use the claimed correspondence to calculate the quark condensate in massless three-flavour QCD and we will compare our prediction with available "data".

\section{Planar equivalence and multi-flavour QCD}

Consider an $S U(N)$ gauge theory with one Dirac fermion $\Psi$ in the twoindex antisymmetric representation and $n_{f}$ Dirac fermions $\chi_{i}$ in the fundamental representation. While at large $N$ the fermions $\chi_{i}$ are suppressed, their presence will affect the finite- $N$ theory. According to planar equivalence, at large $N$ a theory with an antisymmetric fermion is equivalent to $\mathcal{N}=1 \mathrm{SYM}$ in a certain 'common sector'. Assuming indeed that fundamental matter can be neglected at large $N$, QCD-OR' theory interpolates between $S U(3)$ with $N_{f}=n_{f}+1$ flavours and $\mathcal{N}=1 \mathrm{SYM}$ as we increase $N$ from three to infinity. Below we present arguments in favour of the equivalence between large- $N$ SYM and QCD-OR'.

The partition function of $\mathrm{QCD}-\mathrm{OR}^{\prime}$, after integration over the fermions, reads

$$
\begin{aligned}
& \mathcal{Z}_{\mathrm{OR}}^{\prime}=\int D A_{\mu} \exp \left(-S_{\mathrm{YM}}[A]\right) \operatorname{det}(i \not D), \\
& \operatorname{det}(i \not D)=\operatorname{det}\left(i \not \partial+\not A^{a}(T_{\text {anti }}^{a} \oplus \underbrace{T_{\text {fund }}^{a} \oplus T_{\text {fund }}^{a} \oplus \ldots \oplus T_{\text {fund }}^{a}}_{n_{f} \text { times }})\right) .
\end{aligned}
$$

The fermionic determinant can be expressed in terms of Wilson loops [12]. In [13] such an explicit construction was used to prove that 'orientifold field theory' is equivalent to $\mathcal{N}=1 \mathrm{SYM}$ at large $N^{1}$. Let us extend our previous

\footnotetext{
${ }^{1}$ A lattice version of that proof was communicated to us by A. Patella, to appear.
} 
proof to the present case. We first define $\Gamma[A] \equiv \log \operatorname{det}(i \not D)$. Next, we use the results of $[12]$ to express $\Gamma[A]$ in terms of Wilson loops

$$
\begin{aligned}
\Gamma[A] & =-\frac{1}{2} \int_{0}^{\infty} \frac{d T}{T} \\
& \times \int \mathcal{D} x \mathcal{D} \psi \exp \left\{-\int_{\epsilon}^{T} d \tau\left(\frac{1}{2} \dot{x}^{\mu} \dot{x}^{\mu}+\frac{1}{2} \psi^{\mu} \dot{\psi}^{\mu}\right)\right\} \\
& \times \operatorname{Tr} \mathcal{P} \exp \left\{i \int_{0}^{T} d \tau\left(A_{\mu} \dot{x}^{\mu}-\frac{1}{2} \psi^{\mu} F_{\mu \nu} \psi^{\nu}\right)\right\}
\end{aligned}
$$

where $A \equiv A^{a}(T_{\text {anti }}^{a} \oplus \underbrace{T_{\text {fund }}^{a} \oplus T_{\text {fund }}^{a} \oplus \ldots \oplus T_{\text {fund }}^{a}}_{n_{f} \text { times }})$ and similarly $F_{\mu \nu} \equiv F_{\mu \nu}^{a}\left(T_{\text {anti }}^{a} \oplus\right.$ $\underbrace{\left.T_{\text {fund }}^{a} \oplus T_{\text {fund }}^{a} \oplus \ldots \oplus T_{\text {fund }}^{a}\right)}_{n_{f} \text { times }}$. The key ingredient is that a Wilson loop with quarks in the adjoint/antisymmetric representation can be written as

$$
\begin{aligned}
& \operatorname{tr} \exp i \int A^{a} T_{a d j .}^{a}=2\left(\operatorname{tr} U \operatorname{tr} U^{\dagger}-1\right), \\
& \operatorname{tr} \exp i \int A^{a} T_{\text {anti }}^{a}=\frac{1}{2}\left((\operatorname{tr} U)^{2}-\operatorname{tr} U^{2}\right)+\frac{1}{2}\left(\left(\operatorname{tr} U^{\dagger}\right)^{2}-\operatorname{tr}\left(U^{\dagger}\right)^{2}\right),
\end{aligned}
$$

where $\operatorname{tr} U$ denotes a Wilson loop in the fundamental representation.

Moreover, at large- $N$,

$$
\begin{aligned}
& \left(\operatorname{tr} U \operatorname{tr} U^{\dagger}-1\right) \rightarrow \operatorname{tr} U \operatorname{tr} U^{\dagger} \\
& (\operatorname{tr} U)^{2}-\operatorname{tr} U^{2}+\left(\operatorname{tr} U^{\dagger}\right)^{2}-\operatorname{tr}\left(U^{\dagger}\right)^{2} \rightarrow(\operatorname{tr} U)^{2}+\left(\operatorname{tr} U^{\dagger}\right)^{2},
\end{aligned}
$$

since $(\operatorname{tr} U)^{2} \sim O\left(N^{2}\right)$ while $\operatorname{tr} U^{2} \sim O(N)$. Using the above relations (4), one could prove [13] the coincidence of the connected part of Wilson loops correlators in large- $N$ SYM and 'orientifold field theory',

$$
\left\langle W_{a d j .} W_{a d j .} \ldots W_{a d j} .\right\rangle_{\mathrm{conn} .}=\left\langle W_{a n t i} W_{a n t i} \ldots W_{a n t i}\right\rangle_{\mathrm{conn} .} .
$$

The coincidence of the Wilson loops (5) led to the conclusion that the fermionic determinants of SYM and 'orientifold field theory', and hence also the partition functions of the two theories, coincide. 
We can argue that in the present case our previous proof holds by using the following identity

$$
\begin{aligned}
& \operatorname{tr} \exp i \int A^{a}(T_{\text {anti }}^{a} \oplus \underbrace{T_{\text {fund }}^{a} \oplus T_{\text {fund }}^{a} \oplus \ldots \oplus T_{\text {fund }}^{a}}_{n_{f} \text { times }})= \\
& \frac{1}{2}\left((\operatorname{tr} U)^{2}-\operatorname{tr} U^{2}\right)+\frac{1}{2}\left(\left(\operatorname{tr} U^{\dagger}\right)^{2}-\operatorname{tr}\left(U^{\dagger}\right)^{2}\right)+n_{f} \operatorname{tr} U+n_{f} \operatorname{tr} U^{\dagger} .
\end{aligned}
$$

It is obvious that, as expected, the relation with the large- $N$ SUSY theory is not spoiled, since $\operatorname{tr} U$ is $O(N)$, while $(\operatorname{tr} U)^{2}$ is $O\left(N^{2}\right)$. Namely at large $N$ (and $n_{f}$ fixed),

$$
\frac{1}{2}\left((\operatorname{tr} U)^{2}-\operatorname{tr} U^{2}\right)+n_{f} \operatorname{tr} U \rightarrow \frac{1}{2}(\operatorname{tr} U)^{2},
$$

and therefore, as in (5),

$$
\left\langle W_{S Y M} W_{S Y M} \ldots W_{S Y M}\right\rangle_{\mathrm{conn} .}=\left\langle W_{Q C D-O R^{\prime}} W_{Q C D-O R^{\prime}} \ldots W_{Q C D-O R^{\prime}}\right\rangle_{\mathrm{conn} .} .
$$

Moreover, at finite $N$ we can hope for an improved equivalence with respect to the one-flavour case since both $\operatorname{tr} U^{2}$ and $2 n_{f} \operatorname{tr} U$ are $O(N)$, and contribute to (6) with opposite signs. It is interesting to notice that, precisely for $n_{f}=2$, the first non-trivial contribution of the Wilson loop (the one proportional to the YM action) is exactly the same in the QCD-OR' and in SYM theory.

In order to relate the gluino condensate of $\mathcal{N}=1$ Super Yang-Mills with the quark condensate of QCD-OR', we need to couple a source to an appropriate quark bilinear and check for a coincidence at large $N$. The proof of planar equivalence holds also in the presence of such a source. In the next section we discuss the precise form of the matching between bilinear fermion operators in SYM and QCD-OR'.

\section{Matching correlators in QCD-OR' and SYM}

Naively, the presence of extra fermions in the fundamental representation should not spoil the large- $N$ convergence of QCD-OR' towards SYM. However, the situation is actually more subtle. There is no dynamical breaking 
of a continuous chiral symmetry in SYM, since its classical $U_{R}(1)$ symmetry is anomalous. By contrast, QCD-OR', at generic $N^{2}$, has a non-anomalous $S U\left(n_{f}\right)_{L} \otimes S U\left(n_{f}\right)_{R} \otimes U(1)_{V} \otimes U(1)_{A}$ symmetry whose expected dynamical breaking down to $S U\left(n_{f}\right)_{V} \otimes U(1)_{V}$ gives rise to massless Nambu-Golstone $(\mathrm{NG})$ bosons. How can a theory without a mass gap (QCD-OR') be equivalent to a theory with a mass gap (SYM)? Moreover, how best can we match condensates in a theory (SYM) with $N$ discrete vacua with one (QCD-OR') with a continuous vacuum manifold $\left(S^{1} \times S^{3}\right.$ for $\left.n_{f}=2\right)$ ?

The answer that we propose is similar, in spirit, to the one already given $[2,13]$ as a limitation to planar equivalence even at $n_{f}=0$. It was argued that planar equivalence should only hold in a certain bosonic sector of the theory. In the case at hand we will have to further restrict this bosonic sector by excluding, for instance, external NG bosons or sources coupled to them. If the external particles or sources are decoupled from single NGbosons then such bosons can only be pair-produced and this is, generically, a subleading effect. As a result, the two theories should become equivalent within that 'good' sector in the planar limit ${ }^{3}$. The purpose of this section is to find (at least part of) this restricted sector and, in particular, to identify a combination of $\bar{\Psi} \Psi$ and $\bar{\chi} \chi$ that coincides with the gluino condensate of $\mathcal{N}=1$ SYM at large- $N$ and should admit a smooth large- $N$ limit, i.e. one that commutes with sending the masses of the new fermions to zero.

Clearly all the non-abelian flavour currents of QCD-OR' are not in the 'good' sector. Out of the classical $U(1)_{A}$ currents of QCD-OR' ,

$$
J_{\mu 5}^{(\Psi)}=\bar{\Psi} \gamma_{\mu} \gamma_{5} \Psi, \quad J_{\mu 5}^{(\chi)}=\sum_{i=1}^{n_{f}} \bar{\chi}_{i} \gamma_{\mu} \gamma_{5} \chi_{i}
$$

we can construct the non-anomalous conserved current:

$$
J_{\mu 5}^{(c)}=J_{\mu 5}^{(\chi)}-\frac{n_{f}}{N-2} J_{\mu 5}^{(\Psi)} .
$$

For $N=3$ and $n_{f}=2$ this coincides with the usual octet ' $\eta$-meson' current of ordinary three-flavour QCD. We will keep calling $\eta$ the NG boson associated

\footnotetext{
${ }^{2}$ For $N=3$ the symmetry gets enlarged to the standard $S U\left(n_{f}+1\right)_{L} \otimes S U\left(n_{f}+1\right)_{R} \otimes$ $U(1)_{V}$ while at $N=2$ it becomes $S U\left(2 n_{f}\right)$.

${ }^{3}$ Even then it is possible that for certain quantities (e.g. the topological susceptibility) the planar limit $(N \rightarrow \infty)$ and the chiral limit will not commute.
} 
with the spontaneous breaking of the chiral symmetry induced by (10). Let us also define the two 'decay constants'

$$
\left\langle 0\left|J_{\mu 5}^{(\Psi)}\right| \eta\right\rangle=i q_{\mu} F_{\Psi}, \quad\left\langle 0\left|J_{\mu 5}^{(\chi)}\right| \eta\right\rangle=i q_{\mu} F_{\chi} .
$$

The true $\eta$ decay constant $F_{\eta}$ is obviously given by:

$$
\left\langle 0\left|J_{\mu 5}^{(c)}\right| \eta\right\rangle=i q_{\mu} F_{\eta}, \quad F_{\eta}=F_{\chi}-\frac{n_{f}}{N-2} F_{\Psi}
$$

Notice that $F_{\Psi}$ is $O(N)$ while $F_{\chi}$ and $F_{\eta}$ are $O(\sqrt{N})$ as usual.

Now, while the conserved current $J_{\mu 5}^{(c)}$ and thus $F_{\eta}$ are RG-invariant, this is not the case for each individual anomalous current in (9) and for the corresponding decay constants in (11). Indeed the latter are not only scale-dependent but also mix under changes of the renormalization scale. Using RG-invariance of two non-gauge invariant conserved currents, it may be shown that renormalization/mixing take the simple 'factorized' form:

$$
J_{\mu 5 R}^{(i)}=J_{\mu 5 B}^{(i)}+a_{i} \sum_{j} c_{j} J_{\mu 5 B}^{(j)}, \quad F_{R}^{(i)}=F_{B}^{(i)}+a_{i} \sum_{j} c_{j} F_{B}^{(j)},
$$

where here $i=(\Psi, \chi), a_{i}$ are the anomaly coefficients $\left(a_{\Psi}=2(N-2), a_{\chi}=\right.$ $\left.2 n_{F}\right)$, and $c_{i}$ are some renormalization coefficients that one can compute order by order in perturbation theory. (Here, the suffices $R$ and ${ }_{B}$ denote renormalised and bare quantities respectively.)

Let us consider now a linear combination of the currents (9) that decouples from the $\eta$ :

$$
J_{\mu 5}^{(d)}=\frac{B}{F_{\eta}}\left(F_{\chi} J_{\mu 5}^{(\Psi)}-F_{\Psi} J_{\mu 5}^{(\chi)}\right)
$$

where $B$ is a normalisation constant to be fixed in a moment. The anomalous current $J_{\mu 5}^{(d)}$ has two interesting properties:

- One can easily show, using eqs.(13), that $J_{\mu 5}^{(d)}$ is multiplicatively renormalized:

$$
J_{\mu 5 R}^{(d)}=\left(1+a_{i} c_{i}\right) J_{\mu 5 B}^{(d)},
$$

and thus its decoupling is scale-independent.

- The anomaly-coefficient for $J_{\mu 5}^{(d)}$ is just $2 B$, i.e.

$$
\partial^{\mu} J_{\mu 5}^{(d)}=2 B Q
$$


As a result, if we fix $B=N$ the decoupled current $J_{\mu 5}^{(d)}$ will have exactly the same anomaly coefficient as the current $J_{\mu 5}^{(\lambda)}$ in SYM theory. (This also ensures that the two-point function $\left\langle J_{\mu 5}^{(d)} J_{\nu 5}^{(d)}\right\rangle$ is $O\left(N^{2}\right)$ just like $\left\langle J_{\mu 5}^{(\lambda)} J_{\nu 5}^{(\lambda)}\right\rangle$.) It therefore seems compelling to map these two currents into each other in the large- $N$ limit. With this normalisation, we can rewrite $J_{\mu 5}^{(d)}$ in the useful form

$$
J_{\mu 5}^{(d)}=\frac{N}{N-2}\left(J_{\mu 5}^{(\Psi)}-\frac{F_{\Psi}}{F_{\eta}} J_{\mu 5}^{(c)}\right)
$$

The overall normalisation factor $\left(1-\frac{2}{N}\right)^{-1}$ will play a key role below.

Identifying pseudoscalar and scalar operators in the QCD-OR' theory to match with the components $\bar{\lambda} \gamma_{5} \lambda$ and $\bar{\lambda} \lambda$ of the chiral superfield $S^{(\lambda)}$ in SYM is less clear-cut. In particular, the anomalous Ward identities fix the dependence of the condensate $\left\langle S^{(\lambda)}\right\rangle$ on the vacuum angle $\theta$ as $e^{i \theta / N}$, where the factor of $N$ is directly related to the occurrence of the $N$ discrete vacua in SYM. A similar analysis applied to QCD-OR', however, shows that the condensate corresponding to $U=\bar{\Psi} \Psi+i \bar{\Psi} \gamma_{5} \Psi$ has a $\theta$-dependence $\exp \left[i \frac{\theta}{N-2}\left(1+\frac{n_{f}}{N-2} \frac{F_{\Psi}}{F_{\eta}}\right)\right]$ while that for $V=\bar{\chi} \chi+i \bar{\chi} \gamma_{5} \chi$ is $\exp \left[i \frac{\theta}{n_{f}}\left(1-\frac{F_{\chi}}{F_{\eta}}\right)\right]$. The presence of the decay constants in the phase is unusual and reflects the fact that in QCD-OR' a NG boson couples to an anomalous $U(1)$ current. However, recalling the definition (12) of $F_{\eta}$ in terms of $F_{\Psi}$ and $F_{\chi}$, we see that if we construct the combination $T=U^{N-2} \operatorname{det} V$ we obtain an operator whose condensate has the correct $\theta$-dependence, $\langle T\rangle \sim e^{i \theta}$ to match the one of $S^{(\lambda) N}$ in SYM (while dimensions only match at $n_{f}=2$ ). Moreover, we can easily check from the Ward identities that $T$, being a singlet under the conserved $U(1)$, decouples from the NG boson, $\langle 0|T| \eta\rangle=0$.

At first sight it appears that this operator has all the desired properties for its expectation value to match $\left\langle S^{(\lambda) N}\right\rangle$ of SYM. However, planar equivalence holds only for diagrams with a fixed number of external legs, and this is not the case for correlation functions of $T$, which involve diagrams with $O(N)$ external legs. If we had a large- $N$ estimate of the ratio of $\langle T\rangle$ to $\left\langle S^{(\lambda) N}\right\rangle$, each one in units of their respective RG-invariant scales, we could use factorization to arrive at the ratio of the bilinear condensates. Unfortunately, we see no way, at present, to proceed along these lines: it would imply performing a reliable instanton calculation in QCD-OR', similar to the one that gives the exact condensate in SYM. But then we would have already reached our goal without going through the planar correspondence! We shall proceed instead 
in the opposite way: we will discuss planar equivalence (and $1 / N$ corrections to it) directly in terms of bilinear condensates, then use factorization to reconstruct $\langle T\rangle$, and see whether the outcome makes sense in the light of the discussion given above. If this is the case, we shall consider our QCD-OR', SYM condensate relation to be reasonable, and use it at $N=3$ to obtain the quark condensate in QCD.

A decoupled pseudoscalar bilinear is easily obtained by expanding $T$ about its VEV:

$$
P^{(\mathrm{d})}=C\left[\bar{\Psi} \gamma_{5} \Psi+\frac{n_{f}}{(N-2)} \frac{\langle\bar{\Psi} \Psi\rangle}{\langle\bar{\chi} \chi\rangle} \bar{\chi} \gamma_{5} \chi\right]
$$

where $C$ is a normalisation constant which we may take as $1+O(1 / N)$. $P^{(d)}$ is bilinear in the QCD-OR fields $\Psi$ and $\chi$ and maps naturally onto the operator $\bar{\lambda} \gamma_{5} \lambda$ in SYM in the large $N$ limit, where the additional term proportional to $\bar{\chi} \gamma_{5} \chi$ is subleading. We can also verify, directly from the Ward identities, that it decouples from the NG boson, $\left\langle 0\left|P^{(d)}\right| \eta\right\rangle=0$. It therefore satisfies most of the properties we want in order to match to $\bar{\lambda} \gamma_{5} \lambda$. However, we have to recognise that this still represents a compromise, since at $O(1 / N)$ the $\theta$-dependence of the corresponding VEV no longer matches that of $\langle S\rangle$, reflecting the fundamental difference in the vacuum structure of SYM and QCD-OR' at finite $N$.

Using $J_{\mu 5}^{(d)}$ and $P^{(d)}$ to match the corresponding operators in SYM, it is natural to match the SYM condensate ${ }^{4}\langle\lambda \lambda\rangle \equiv\left\langle\lambda_{\alpha}^{a} \lambda^{a, \alpha}\right\rangle$ with the condensate $\left\langle S^{(d)}\right\rangle$ arising in the anomalous Ward identity

$$
\partial_{\mu}\left\langle J_{\mu 5}^{(d)} P^{(d)}\right\rangle=2 N\left\langle Q P^{(d)}\right\rangle+2\left\langle S^{(d)}\right\rangle
$$

Recalling the form (17) of the decoupled current, we readily find

$$
S^{(d)}=C\left(\frac{N}{N-2}\right)\left[\bar{\Psi} \Psi+\frac{n_{f}}{N-2} \frac{F_{\Psi}}{F_{\eta}}\left(\bar{\Psi} \Psi-\frac{\langle\bar{\Psi} \Psi\rangle}{\langle\bar{\chi} \chi\rangle} \bar{\chi} \chi\right)\right],
$$

where we stress that the second term in the square brackets, which is the variation of $P^{(d)}$ corresponding to the conserved current, necessarily has a vanishing $\mathrm{VEV}$. We see, therefore, that although the operator $S^{(d)}$ is a linear combination of $\bar{\Psi} \Psi$ and $\bar{\chi} \chi$, the appropriate condensate $\left\langle S^{(d)}\right\rangle$ in QCD-OR'

\footnotetext{
${ }^{4}$ Notice that from this point on, we revert to using Weyl notation for the gluino.
} 
to be matched to $\langle\lambda \lambda\rangle$ in SYM is simply $\langle\bar{\Psi} \Psi\rangle$ itself, up to an important normalisation. The overall factor $\left(1-\frac{2}{N}\right)^{-1}$ in $(20)$ is just the normalisation factor defining the decoupled current $J_{\mu 5}^{(d)}$. It has a clear physical significance related to the vanishing of the condensate $\langle\bar{\Psi} \Psi\rangle$ at $N=2$, where the antisymmetric representation fermion becomes a colour singlet. Unfortunately, we have no such clear argument for the normalisation factor $C$ for $P^{(d)}$.

Consider therefore, as in [4], the ratio of ratios

$$
K\left(1 / N, n_{f}\right) \equiv \frac{\langle\bar{\Psi} \Psi\rangle_{R G I} / \Lambda_{O R^{\prime}}^{3}}{\langle\lambda \lambda\rangle / \Lambda_{S Y M}^{3}}=\left(1-\frac{2}{N}\right) \frac{1}{C} \frac{\left\langle S^{(d)}\right\rangle_{R G I} / \Lambda_{O R^{\prime}}^{3}}{\langle\lambda \lambda\rangle / \Lambda_{S Y M}^{3}}
$$

where planar equivalence and the above identification of $\left\langle S^{(d)}\right\rangle$ with the gluino condensate $\langle\lambda \lambda\rangle$ ensures that $K=1+O(1 / N)$. As in ref.[4], it is convenient to write

$$
K\left(1 / N, n_{f}\right)=\left(1-\frac{2}{N}\right) K_{*}\left(1 / N, n_{f}\right)
$$

where we explicitly display the normalisation factor arising from $J^{(d)}$ but absorb the unknown normalisation $C$ from $P^{(d)}$ into $K_{*}$. We will then argue on more general grounds how the $O(1 / N)$ corrections to $K_{*}$ may be constrained.

Before we match the condensates of the two theories we wish to explain our conventions. Our slightly unconventional two-loop $\Lambda$ parameters are defined in terms of the renormalization scale $\mu$ and the 't Hooft coupling $\lambda(\mu) \equiv \frac{\alpha(\mu) N}{2 \pi}$ by:

$$
\Lambda=\mu(\lambda(\mu))^{-\frac{\beta_{1}}{\beta_{0}^{2}}} \exp \left(-\frac{N}{\beta_{0} \lambda(\mu)}\right)
$$

where $\beta_{0}$ and $\beta_{1}$ are the one and two-loop beta function coefficients, respectively (for discussion of the subtraction scheme see ref.[4]). We also use the following standard convention for both QCD-OR' and SYM:

$$
\mathcal{L}=-\frac{1}{2 g^{2}} \operatorname{tr} F^{2}+i \bar{q} \not D q
$$

( $q$ here can be either $\Psi, \chi$, or $\lambda$ ) and define a renormalization group invariant quark condensate as follows

$$
\langle\bar{q} q\rangle_{R G I} \equiv\left\langle(\lambda)^{\frac{\gamma}{\beta_{0}}} \bar{q} q\right\rangle
$$


where $\gamma$ is the one-loop anomalous dimension coefficient for the fermion bilinear. Note that for SYM, $\gamma=\beta_{0}=3 N$. In these conventions [1]:

$$
\frac{\langle\lambda \lambda\rangle_{R G I}}{\Lambda_{S Y M}^{3}}=-\frac{N^{2}}{2 \pi^{2}}
$$

Putting all this together, we end up with:

$$
\frac{\langle\bar{\Psi} \Psi\rangle_{R G I}}{\Lambda_{O R^{\prime}}^{3}}=-\frac{N^{2}}{2 \pi^{2}}\left(1-\frac{2}{N}\right) K_{*}\left(1 / N, n_{f}\right)
$$

Before proceeding to estimate $K_{*}$ let us check whether eq.(27) gives something reasonable for the corresponding ratio of $\langle T\rangle$ and $\left\langle S^{(\lambda) N}\right\rangle$. Let us define:

$$
\frac{\langle\bar{\chi} \chi\rangle_{R G I}}{\Lambda_{O R^{\prime}}^{3}}=-\frac{N}{2 \pi^{2}} \tilde{K}\left(1 / N, n_{f}\right)
$$

with $\tilde{K}\left(1 / 3, n_{f}\right)=K_{*}\left(1 / 3, n_{f}\right)$ but where $\tilde{K}\left(0, n_{f}\right)$ need not necessarily be 1. Using factorization ${ }^{5}$ we would predict:

$$
\frac{\langle T\rangle / \Lambda_{O R^{\prime}}^{3\left(N+n_{f}-2\right)}}{\left\langle S^{(\lambda) N}\right\rangle / \Lambda_{S Y M}^{3 N}}=K_{*}^{N-2} \tilde{K}^{n_{f}} N^{n_{f}} \frac{(N-2)^{N-2}}{N^{N+2}} \sim \frac{K^{N-2} \tilde{K}^{n_{f}} N^{n_{f}}}{e^{2} N^{2}} \frac{(N-2) !}{N !}
$$

Is this result compatible with the assumption $K_{*}, \tilde{K} \sim 1$ at large $N$ ? We believe that it is. In particular, an $(N-2)$ ! is known to originate from distributing the $2 N$ gluino zero modes among the $2 N$ fermions appearing in the correlator $\left\langle S^{(\lambda) N}\right\rangle[14]$. In the QCD-OR' case a similar argument applied to $\langle T\rangle$ would naturally produce an $(N-4)$ ! corresponding to the smaller number of $\Psi$ zero modes contributing to that correlator. Notice that the number of fermionic zero modes is independent of the single-instanton approximation. It depends only on the topological charge of the gauge configurations contributing to $\left\langle S^{(\lambda) N}\right\rangle$ and $\langle T\rangle$.

We finally turn to estimating $K_{*}$ and $\tilde{K}$. Up to order $1 / N^{2}, K$ has the general form

$$
K\left(1 / N, n_{f}\right)=1+\frac{\alpha+\beta n_{f}}{N}+\frac{\gamma+\delta n_{f}+\epsilon n_{f}^{2}}{N^{2}}
$$

\footnotetext{
${ }^{5}$ Factorization is exact at large $N$ but it also expected to be a good approximation at finite $N$ provided the bilinear condensate is dynamically favoured over multifermion condensates.
} 
Implementing the condition that $K$ vanishes at $N=2$ for all $n_{f}$ imposes three constraints on the parameters $\alpha, \ldots \epsilon$, including $\epsilon=0$. At this order, we can therefore approximate $K_{*}$ by

$$
K_{*}\left(1 / N, n_{f}\right)=1+\frac{\alpha+2}{N}+\frac{\beta n_{f}}{N},
$$

In order to estimate the size of $\alpha$ and $\beta$ we use the following argument. The QCD-OR' theory loses asymptotic freedom and becomes IR free when

$$
n_{f}>\frac{9}{2} N+2 \equiv n_{A F}
$$

It is clear that all condensates should vanish for such large values of $n_{f}$. This is implemented in (27) by the vanishing of $\Lambda_{O R^{\prime}}$ when $\beta_{0}=0$ (see next section). On the other hand, even in the range with $n_{f}<n_{A F}$, the theory, while still asymptotically free, develops a non-trivial IR fixed point [15]. It is reasonable to parametrize the lower limit $n_{C W}$ of such a 'conformal window' $\left(n_{C W}<n_{f}<n_{A F}\right)$ as:

$$
n_{C W}=a N+b,
$$

and to assume that all condensates go to zero as $n_{f} \rightarrow n_{C W}$. We implement this condition directly in $K_{*}\left(1 / N, n_{f}\right)$.

Within these approximations, we easily find the following unique solution for $K_{*}$ and $\tilde{K}$ :

$$
K_{*}=\tilde{K}=\left(1-\frac{1}{a N}\left(n_{f}-b\right)\right) .
$$

which shows clearly that the value of the condensate decreases with an increasing number of flavours.

Together with the factor $\left(1-\frac{2}{N}\right)$ in $(22)$, this gives an expression for $K$ which implements the two constraints we have on the condensate - vanishing at $N=2$ where the antisymmetric representation degenerates to a colour singlet, and the existence of a conformal window - along with planar equivalence, which fixes the large- $N$ limit $K\left(0, n_{f}\right)=1$. For fixed $n_{f}$, we therefore have information on $K\left(1 / N, n_{f}\right)$ at three values of $N$. Combining (22) with (34) fits this 'data' with a quadratic form, which we may expect to be a good approximation. 
Expectations $^{6}$ for $a$ and $b$ are around $2 \pm 1$ [16]. Inserting $a=b=2$ in (34), our final result for the three-flavour QCD condensate, $N=3, n_{f}=2$, becomes:

$$
\frac{\langle\bar{\Psi} \Psi\rangle_{R G I}}{\Lambda_{O R^{\prime}}^{3}}=-\frac{9}{2 \pi^{2}} K(1 / 3,2) \simeq-0.15 \pm 0.05
$$

The error arises from the uncertainty involved in the truncation of $K$ and the values of $a, b$ determining the conformal window. Notice, however, that the latter is minimised for $n_{f} \simeq b \simeq 2$. We conservatively estimate the total error as $30 \%$.

In the following section, after giving some more arguments why the case $n_{f}=2$ should be particularly favourable, we turn to a discussion of the comparison of our prediction with real and Monte-Carlo data.

\section{Quark condensate in massless three-flavour QCD}

So far we have considered QCD-OR' for general $n_{f}$, which reduces to multi-flavour massless QCD at $N=3$. We now specialise to $n_{f}=2$ and estimate the quark condensate in three-flavour QCD. We restrict ourselves to three flavours, since it is both the most interesting case from the phenomenological point of view and since our approximation is expected to be optimal when $n_{f}=2$. The reason is the following: as we shall see, the calculation of the condensate is sensitive to the value of the one and two-loop

\footnotetext{
${ }^{6}$ There is no easy criterion for determining the lower boundary of the conformal window. However, in ref.[16], Gardi and Grunberg discuss one criterion, based on the analyticity properties of the coupling as determined by the beta function, which is simple to estimate. With our conventions (see section 4 ), this condition is $-\beta_{0}^{2} / 2 \beta_{1}>1$. For QCD with $N_{f}$ fundamental flavours, this gives $N_{f C W} \simeq 3.2 N$. This is higher than some lattice estimates [17], which give $N_{f C W} \simeq 7$ for $N=3$. Nevertheless, we may use the analyticity criterion to get at least a rough estimate of $n_{C W}$ in QCD-OR'. For QCD-OR', with one fermion in the antisymmetric representation and $n_{f}$ fundamentals, the beta function coefficients are$$
\beta_{0}=3 N-\frac{2}{3}\left(n_{f}-2\right) \quad \beta_{1}=3 N^{2}+2 N-\frac{3}{N}-\left(\frac{13}{6} N-\frac{1}{2 N}\right)\left(n_{f}-2\right)
$$

We can then show that the condition $-2 \beta_{1} \simeq \beta_{0}^{2}$ is approximately satisfied for $N \geq 3$ by $n_{f}=a N+b$ with $a \simeq 2, b \simeq 2.6$.
} 
beta function coefficients. For $n_{f}=2$ the one-loop beta function of QCD$\mathrm{OR}^{\prime}$ is $\beta_{0}=3 N$, exactly as in $\mathcal{N}=1 \mathrm{SYM}$. The two-loop beta function is $\beta_{1}=3 N^{2}\left(1+2 /(3 N)-1 / N^{3}\right)$, which is very close to the value of the SUSY theory $\left(\beta_{1}=3 N^{2}\right)$. The underlying reason is as follows: the Dynkin index of a certain representation reflects the number of degrees of freedom that run in the vacuum polarization loop. For the adjoint it is $N$, while for the antisymmetric representation it is $\frac{1}{2}(N-2)$ and for the fundamental representation it is $\frac{1}{2}$. Since we have a Dirac fermion in the antisymmetric and two Dirac fermions in the fundamental we get $(N-2)+2=N$. In this sense, the physical degrees of freedom in our model are the same as those of an adjoint Majorana fermion. Similarly, we saw in section 2 that the expectation value of a Wilson loop in QCD-OR' might approach the expectation value of a Wilson loop in SYM with rather mild $1 / N$ corrections when $n_{f}=2$.

Let us proceed to the calculation of the quark condensate. Our conclusion from the last section is that for $S U(3)$,

$$
\langle\bar{\Psi} \Psi\rangle_{R G I} \simeq-\frac{3}{2 \pi^{2}} \Lambda_{O R^{\prime}}^{3}
$$

In order to compare the value of the condensate to lattice predictions, it is useful to write (37) at an arbitrary scale $\mu$. Using our previously introduced definitions gives:

$$
\langle\bar{\Psi} \Psi\rangle_{\mu}=-\frac{3}{2 \pi^{2}} \mu^{3}(\lambda(\mu))^{-\frac{\gamma}{\beta_{0}}-\frac{3 \beta_{1}}{\beta_{0}^{2}}} \exp \left(-\frac{9}{\beta_{0} \lambda(\mu)}\right) .
$$

For three-flavour QCD the relevant values are $\beta_{0}=9, \beta_{1}=32, \gamma=4$.

In order to calculate the condensate (38) we need to know the value of the 't Hooft coupling $\lambda$ at a scale $\mu$ that we choose to be $\mu=2 \mathrm{GeV}$. The Particle Data Group [18] quotes $\alpha_{s}(2 \mathrm{GeV})=0.31 \pm 0.01$ which corresponds to $\lambda(2 \mathrm{GeV})=0.148 \pm 0.010$. We therefore choose to plot the function

$$
\frac{\langle\bar{\Psi} \Psi\rangle_{2 \mathrm{GeV}}}{\mathrm{GeV}^{3}}=-\frac{3}{2 \pi^{2}} 2^{3} \lambda^{-\frac{44}{27}} \exp \left(-\frac{1}{\lambda}\right)
$$

in a range of $\lambda$. The results are presented in fig.(1). 


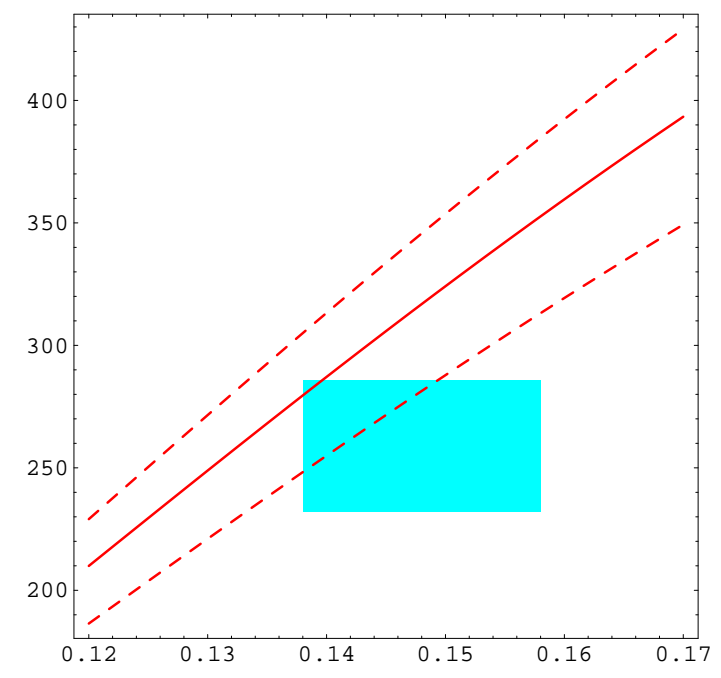

Figure 1: The quark condensate expressed as $-(y \mathrm{MeV})^{3}$ as a function of the 't Hooft coupling $\lambda$. The $\pm 1 \sigma$ range of the coupling, $0.138<\lambda<0.158$ and the lattice estimate $-(259 \pm 27 \mathrm{MeV})^{3}$ define the shaded region.

The solid line in fig.1 shows the condensate $\langle\bar{\Psi} \Psi\rangle_{2} \mathrm{GeV}$ evaluated from (39) as a function of the 't Hooft coupling $\lambda(2 \mathrm{GeV})$. With the central value $\lambda=0.148$, we find

$$
\langle\bar{\Psi} \Psi\rangle_{2 \mathrm{GeV}}=-(317 \pm 30 \pm 36 \mathrm{MeV})^{3}
$$

Here, we have included an error $\pm 30 \%$ in the condensate due to the uncertainties in estimating $K$ discussed in the previous section. This is indicated in the plot by the dashed lines. The second error shown in (40) reflects the $\pm 1 \sigma$ range of the experimentally determined coupling.

We can compare this result with estimates from lattice QCD. Up to now, these have mostly been performed in the quenched approximation with overlap fermions, with the condensate either evaluated directly or deduced from mass ratios using the Dashen-Gell-Mann-Oakes-Renner (DGMOR) relation. Two recent results in this category are those of Giusti et al. [19] who find $-(267 \pm 16 \mathrm{MeV})^{3}$ and Wennekers and Wittig [20] who quote $-(285 \pm 9 \mathrm{MeV})^{3}$. A recent analysis using dynamical fermions has been 
given by McNeile [21] using MILC data for mass ratios together with the DGMOR relation. He finds $-(259 \pm 27 \mathrm{MeV})^{3}$. (Ref.[21] also contains a useful compendium of previous lattice estimates.) This is the result shown in the shaded band in fig.1, extended across the $1 \sigma$ range of $\lambda(2 \mathrm{GeV})$.

It is apparent from these comparisons that our method tends to slightly over-estimate the condensate compared with the lattice evaluations, at least within the $1 \sigma$ range of $\alpha_{s}(2 \mathrm{GeV})$. A lower coupling would obviously ease the agreement. It is also clear that both methods still have significant uncertainties. However, bearing this in mind, the degree of agreement is extremely encouraging, especially when one remembers that we are calculating a non-perturbative quantity in QCD from first principles using entirely analytic methods. In conclusion, we find this result very promising for the programme of exploiting our knowledge of exact results in supersymmetric gauge theories to calculate non-perturbative quantities in QCD itself. 


\section{ACKNOWLEDGMENTS}

A.A. and G.V. would like to thank L. Del-Debbio for very useful discussions. We thank M. Shifman for collaboration in the early stages of this work and for many fruitful discussions and comments. A.A. is supported by the PPARC advanced fellowship award.

\section{References}

[1] The gluino condensate in supersymmetric gluodynamics was first conjectured, on the basis of the value of his index, by E. Witten, Nucl. Phys. B 202, 253 (1982). It was confirmed in an effective Lagrangian approach by G. Veneziano and S. Yankielowicz, Phys. Lett. B 113, 231 (1982), and exactly calculated (by using holomorphy and analytic continuations in mass parameters) in M. A. Shifman and A. I. Vainshtein, Nucl. Phys. B 296, 445 (1988).

[2] A. Armoni, M. Shifman and G. Veneziano, Nucl. Phys. B 667, 170 (2003) [hep-th/0302163].

[3] A. Armoni, M. Shifman and G. Veneziano, Phys. Rev. Lett. 91, 191601 (2003) [hep-th/0307097].

[4] A. Armoni, M. Shifman and G. Veneziano, Phys. Lett. B 579, 384 (2004) [hep-th/0309013].

[5] A. Armoni, M. Shifman and G. Veneziano, "From super-Yang-Mills theory to QCD: Planar equivalence and its implications," hep-th/0403071.

[6] F. Sannino and M. Shifman, Phys. Rev. D 69, 125004 (2004) [hepth/0309252].

[7] D. K. Hong, S. D. H. Hsu and F. Sannino, Phys. Lett. B 597, 89 (2004) [hep-ph/0406200].

[8] A. Feo, P. Merlatti and F. Sannino, "Information on the super YangMills spectrum," hep-th/0408214. 
[9] P. Di Vecchia, A. Liccardo, R. Marotta and F. Pezzella, "Brane-inspired orientifold field theories," hep-th/0407038.

[10] A. Armoni and E. Imeroni, "Predictions for orientifold field theories from type 0' string theory," hep-th/0508107.

[11] E. Corrigan and P. Ramond, Phys. Lett. B 87, 73 (1979).

[12] R. Casalbuoni, J. Gomis and G. Longhi, Nuovo Cim. 24A , 249 (1974); R. Casalbuoni, Nuovo. Cim. 33A, 389 (1976); A. Barducci, F. Bordi and R. Casalbuoni, Nuovo Cim. 64B, 287 (1981); A. Barducci, R. Casalbuoni and L. Lusanna, Nucl. Phys. B 180, 141 (1981); L. Brink, P. Di Vecchia and P. S. Howe, Nucl. Phys. B 118, 76 (1977); M. J. Strassler, Nucl. Phys. B 385, 145 (1992) [hep-ph/9205205].

[13] A. Armoni, M. Shifman and G. Veneziano, Phys. Rev. D 71, 045015 (2005) [hep-th/0412203].

[14] D. Amati et al. Phys. Rep. 162 (1988) 169.

[15] T. Banks and A. Zaks, Nucl. Phys. B 196 (1982) 189.

[16] E. Gardi and G. Grunberg, JHEP 9903, 024 (1999) [hep-th/9810192].

[17] Y. Iwasaki, K. Kanaya, S. Kaya, S. Sakai and T. Yoshie, Phys. Rev. D 69 (2004) 014507 [hep-lat/0309159].

[18] I. Hinchliffe, 'Quantum Chromodynamics', Particle Data Group, Phys. Lett. B 592, 1 (2004)

[19] L. Giusti, C. Hoelbling and C. Rebbi, Phys. Rev. D 64, 114508 (2001) [Erratum-ibid. D 65, 079903 (2002)] [hep-lat/0108007].

[20] J. Wennekers and H. Wittig, JHEP 0509 (2005) 059 [hep-lat/0507026].

[21] C. McNeile, Phys. Lett. B 619, 124 (2005) [hep-lat/0504006]. 